Research Article

\title{
The Slow Spinning Motion of a Rigid Body in Newtonian Field and External Torque
}

\author{
A. I. Ismail iD ${ }^{1,2}$ \\ ${ }^{1}$ Department of Mechanics, College of Engineering and Islamic Architecture, Umm Al-Qura University, P.O. Box 5555, \\ Mecca, Saudi Arabia \\ ${ }^{2}$ Department of Mathematics, Faculty of Science, Tanta University, P.O. Box 31527, Tanta, Egypt
}

Correspondence should be addressed to A. I. Ismail; aiismail@uqu.edu.sa

Received 27 June 2020; Revised 7 August 2020; Accepted 12 August 2020; Published 15 October 2020

Guest Editor: Gang Zhang

Copyright $(2020$ A. I. Ismail. This is an open access article distributed under the Creative Commons Attribution License, which permits unrestricted use, distribution, and reproduction in any medium, provided the original work is properly cited.

\begin{abstract}
In this paper, the problem of the slow spinning motion of a rigid body about a point $O$, being fixed in space, in the presence of the Newtonian force field and external torque is considered. We achieve the slow spin by giving the body slow rotation with a sufficiently small angular velocity component $r_{0}$ about the moving $z$-axis. We obtain the periodic solutions in a new domain of the angular velocity vector component $r_{0} \longrightarrow 0$, define a large parameter proportional to $1 / r_{0}$, and use the technique of the large parameter for solving this problem. Geometric interpretations of motions will be illustrated. Comparison of the results with the previous works is considered. A discussion of obtained solutions and results is presented.
\end{abstract}

\section{Introduction}

In [1], the problem of rigid body dynamics is considered. The author in [2] gave important space applications to this problem. In [3], the authors presented valuable and important studies for the evolution of motions of a rigid body about its mass center. In [4], the authors introduced a new procedure for solving Euler-Poisson equations (of a rotatory rigid body over a fixed point). The author in [5] constructed periodic solutions for Euler-Poisson equations utilizing power series expansion containing a small parameter proportional to the inverse of sufficiently high angular velocity component. In [6], the author studied many perturbation techniques for solving the linear and nonlinear systems of ordinary and partial differential equations such as Poincare's method, KBM method, Poincaré-Lindstedt method, and multiple scales method. The authors in [7] studied new types of integrable twovariable systems with quartic second integrals. The study in [8] presented the motion for the rigid body in the presence of a gyrostatic momentum in cases of external effects and without external effects. The author considered the fast spin motion of a rigid body and achieved a small parameter proportional to the inverse of high angular components about the $z$-axis. The author applies the small parameter of Poincare's method for solving this problem. In [9], the author investigated the motion over the fixed point $O$ of a fast spinning heavy solid in a uniform gravity field (the classical problem). He assumed fast spinning of the body, achieved a small parameter, and used Poincare's method for the solution. In all previous works, the rotary motion for a fast-spinning body with gyro moments was studied. Initially, the authors assumed that the body rotates with a sufficiently large angular velocity component $r_{o}$ about the moving $z$-axis which moves with the body. The authors achieved a small parameter proportional to $1 / r_{0}$ and used the small parameter technique to solve the considered problems in the domain $\left(t, r_{o} \longrightarrow \infty, \varepsilon \longrightarrow 0\right)$. The fact of slow motion of that body which must be achieved on a new parameter named the large parameter and must be solved using a new procedure named the large parameter technique was not considered, although this motion saves high energy given at the initial moment of the body and can solve the problem in a new domain $\left(t, r_{o} \longrightarrow 0, \varepsilon \longrightarrow \infty\right)$. 


\section{Equations of Motion and Change of Variables}

Consider a rigid body of mass $M$ [10], with arbitrary ellipsoid of inertia surface, rotating about a fixed point $O$ in the presence of the Newtonian force field $O_{1}$ under the influence of the external torque vector about the moving axes $\underline{\ell}=\ell_{1} \hat{i}+\ell_{2} \hat{j}+\ell_{3} \underline{\hat{k}}$. Let the attracting center $O_{1}$ lie on the $Z$-axis which is fixed in space. Let the element $\mathrm{d} m$ lie on the body at the point $p(x, y, z)$ and have a position vector $\rho$ from $O$ and a position vector $\underline{r}$ from $O_{1}$. Equations of motion and their first integrals are achieved and solved with a sufficiently large parameter proportional to $1 / r_{0}$, where $r_{0}$ is sufficiently small. We deduce the system of equations of motion and their first integrals of the considered problem and use the large parameter method for solving it.

The differential equations of motion and their first integrals are obtained [10]. Let $\underline{h}_{o}$ be the angular momentum vector which rotates in space at the same angular velocity $\underline{\omega}$ of the rigid body and $\underline{\underline{k}}=\left(\gamma, \gamma^{\prime}, \gamma^{\prime \prime}\right)$ be the unit vector fixed in space in the direction of the downward $Z$-axis, so

$$
\begin{aligned}
& \underline{h}_{o}=\left(A p+\ell_{1}\right) \underline{\hat{i}}+\left(B q+\ell_{2}\right) \underline{\hat{j}}+\left(C r+\ell_{3}\right) \underline{\hat{k}}, \\
& \underline{\omega}=p \underline{\hat{i}}+q \underline{\hat{j}}+r \underline{\hat{k}},
\end{aligned}
$$

where $A, B$, and $C$ are the body's principal moments of inertia in the moving frame. The six nonlinear equations of motion for this case are obtained in the following form:

$$
\begin{aligned}
\frac{\mathrm{d} \underline{h}_{o}}{\mathrm{~d} t}= & {\left[A \frac{\partial p}{\partial t}+(C-B) q r\right] \widehat{i}+\left[B \frac{\partial q}{\partial t}+(A-C) p r\right] \underline{j} } \\
& +\left[C \frac{\partial r}{\partial t}+(B-A) p q\right] \underline{\hat{k}},
\end{aligned}
$$$$
\frac{\mathrm{d} \underline{\widehat{K}}}{\mathrm{~d} t}=\frac{\partial \underline{\widehat{K}}}{\partial t}+\underline{\omega} \wedge \underline{\widehat{K}}=\underline{0} .
$$
follows:

These equations have three first integrals named as

(a) The Jacobi-integral

$$
T+V=\text { const, }
$$

where $T$ is the kinetic energy of the body and $V$ is the potential one.

(b) The angular momentum integral

$$
\underline{h}_{0} \cdot \underline{\widehat{K}}=\text { const. }
$$

(c) The geometric integral

$$
\underline{\widehat{K}} \cdot \underline{\widehat{K}}=1 .
$$

Equations (3) and (4) are nonlinear differential equations for the motion of a rigid body around a fixed point in the field of Newtonian force with the presence of rotary torque vector $\underline{\ell}\left(\ell_{1}, \ell_{2}, \ell_{3}\right)$, around the $x$-axis, the $y$-axis, and the $z$-axis, respectively.

These equations are of first order in unknown variables $p, q, r, \gamma, \gamma^{\prime}$, and $\gamma^{\prime \prime}$. The quantities $A, B, C, \ell_{1}, \ell_{2}$, and $\ell_{3}$ are constants. The integration of such equations gives the solutions $p, q, r, \gamma, \gamma^{\prime}$, and $\gamma^{\prime \prime}$ as functions in time $t$ and the rigid body parameters.

The equations of motion for a coherent object around a fixed point in the asymmetric attraction field $[5,9]$ and their three initial integrals result as special cases from equations (3), (4), (5), (6), and (7).

Let $\left(x_{0}, y_{0}, z_{0}\right)$ be the center of mass in the moving coordinate system $(O x y z) ; R$ is the distance from the fixed point $O$ to the attracting center $O_{1} ; p_{0}, q_{0}, r_{0}, \gamma_{0}, \gamma_{0}^{\prime}$, and $\gamma_{0}^{\prime \prime}$ are the initial values of the corresponding variables. Initially, let the body rotate about the $z$-axis with a sufficiently small angular velocity component $r_{0}$ such that the $z$-axis makes an angle $\theta_{0} \neq 0.5 n \pi(n=0,1,2, \ldots)$ with $Z$-axis being fixed in space.

Without a loss of generality, we choose the positive $z$ axis, and the $x$-axis does not make an obtuse angle with $Z$ axis. According to this restriction, we obtain [9]

$$
\gamma_{0} \geq 0,0<\gamma_{0}^{\prime \prime}<1 \text {. }
$$

Assume the parameters as follows:

$$
\begin{aligned}
a & =\frac{A}{C}, \quad(a b), \\
c^{2} & =\frac{M g \ell}{C}, \\
\varepsilon & =\frac{c \sqrt{\gamma_{0}^{\prime \prime}}}{r_{0}}, \\
x_{0} & =\ell x_{0}^{\prime},\left(x_{o} y_{o} z_{o}\right), \\
\ell^{2} & =x_{0}^{2}+y_{0}^{2}+z_{0}^{2},
\end{aligned}
$$

where $\varepsilon$ is large since $r_{0}$ is small and symbols such as (abc) mean cyclic permutations and indicate equations which are omitted.

Introducing new variables as follows:

$$
\begin{aligned}
& p=c \sqrt{\gamma_{0}^{\prime \prime}} p_{1}, \\
& r=r_{0} r_{1}, \\
& \gamma=\gamma_{0}^{\prime \prime} \gamma_{1}, \quad\left(p q, \gamma \gamma^{\prime} \gamma^{\prime \prime}\right), \\
& k=\frac{3 g}{R} c^{-2}, \\
& t=\frac{\tau}{r_{0}} .
\end{aligned}
$$


Substituting equation (10) into equations (3) to (7) when $\ell_{1}=\ell_{2}=0$, we obtain

$$
\begin{aligned}
& \dot{p}_{1}+A_{1} q_{1} r_{1}+A^{-1} r_{0}^{-1} q_{1} \ell_{3}=\varepsilon^{-1} a^{-1}\left(y_{0}^{\prime} \gamma_{1}^{\prime \prime}-z_{0}^{\prime} \gamma_{1}^{\prime}+k a A_{1} \gamma_{1}^{\prime} \gamma_{1}^{\prime \prime}\right) \\
& \dot{q}_{1}+B_{1} p_{1} r_{1}-B^{-1} r_{0}^{-1} p_{1} \ell_{3}=\varepsilon^{-1} b^{-1}\left(z_{0}^{\prime} \gamma_{1}-x_{0}^{\prime} \gamma_{1}^{\prime \prime}+k b B_{1} \gamma_{1} \gamma_{1}^{\prime \prime}\right) \\
& \dot{r}_{1}=\varepsilon^{-2}\left(-C_{1} p_{1} q_{1}+x_{0}^{\prime} \gamma_{1}^{\prime}-y_{0}^{\prime} \gamma_{1}+k C_{1} \gamma_{1} \gamma_{1}^{\prime}\right), \\
& \dot{\gamma}_{1}=r_{1} \gamma_{1}^{\prime}-\varepsilon^{-1} q_{1} \gamma_{1}^{\prime \prime}, \\
& \dot{\gamma}_{1}^{\prime}=\varepsilon^{-1} p_{1} \gamma_{1}^{\prime \prime}-r_{1} \gamma_{1}, \\
& \dot{\gamma}_{1}^{\prime \prime}=\varepsilon^{-1}\left(q_{1} \gamma_{1}-p_{1} \gamma_{1}^{\prime}\right), \quad\left(. \equiv \frac{\mathrm{d}}{\mathrm{d} \tau}\right) \\
& r_{1}^{2}=1+\varepsilon^{-2} S_{1}, \\
& r_{1} \gamma_{1}^{\prime \prime}=1+\varepsilon^{-1} S_{2}, \\
& \gamma_{1}^{2}+\gamma_{1}^{\prime 2}+\gamma_{1}^{\prime \prime 2}=\left(\gamma_{0}^{\prime \prime}\right)^{-2}
\end{aligned}
$$

where

$$
\begin{aligned}
S_{1}= & a\left(p_{10}^{2}-p_{1}^{2}\right)+b\left(q_{10}^{2}-q_{1}^{2}\right) \\
& -2\left[x_{0}^{\prime}\left(\gamma_{10}-\gamma_{1}\right)+y_{0}^{\prime}\left(\gamma_{10}^{\prime}-\gamma_{1}^{\prime}\right)+z_{0}^{\prime}\left(1-\gamma_{1}^{\prime \prime}\right)\right] \\
& +k\left[a\left(\gamma_{10}^{2}-\gamma_{1}^{2}\right)+b\left(\gamma_{10}^{\prime 2}-\gamma_{1}^{\prime 2}\right)+\left(1-\gamma_{1}^{\prime \prime 2}\right)\right]
\end{aligned}
$$

$$
S_{2}=a\left(p_{10} \gamma_{10}-p_{1} \gamma_{1}\right)+b\left(q_{10} \gamma_{10}^{\prime}-q_{1} \gamma_{1}^{\prime}\right)+\frac{\left(1-\gamma_{1}^{\prime \prime}\right) \ell_{3}}{\left(C c \sqrt{\gamma_{0}^{\prime \prime}}\right)}
$$

\section{Reduction of the Equations of Motion to a Quasi-Linear Autonomous System}

In this section, we reduce the equations of motion to a quasilinear autonomous system [11]. From equations (17) and (18), we obtain

$$
\begin{aligned}
& r_{1}=1+0.5 \varepsilon^{-2}\left[S_{1}+2 z_{0}^{\prime}\left(1-\gamma_{1}^{\prime \prime}\right)-k\left(1-\gamma_{1}^{\prime \prime 2}\right)\right]+\cdots \\
& \gamma_{1}^{\prime \prime}=1+\varepsilon^{-1} S_{2}-0.5 \varepsilon^{-2}\left[S_{1}+2 z_{0}^{\prime}\left(1-\gamma_{1}^{\prime \prime}\right)-k\left(1-\gamma_{1}^{\prime \prime}\right)\right]+\cdots
\end{aligned}
$$

Differentiating equations (11) and (14) and using (21), one obtains

$$
\begin{aligned}
p_{1}+\omega^{\prime 2} p_{1}= & \varepsilon^{-1}\left\{z_{0}^{\prime}\left(a^{-1}-A_{1} b^{-1}\right) \gamma_{1}+A_{1} b^{-1} x_{0}^{\prime}+k\left(\omega^{2}-A_{1}\right) \gamma_{1}+\left[b^{-1}\left(x_{0}^{\prime}-z_{0}^{\prime} \gamma_{1}\right)-k B_{1} \gamma_{1}\right] A^{-1} r_{0}^{-1} \ell_{3}\right\} \\
& +\varepsilon^{-2}\left\{\left[-\omega^{2} p_{1} S_{1}+A_{1} b^{-1} x_{0}^{\prime} S_{2}+A_{1} C_{1} p_{1} q_{1}^{2}-A_{1} q_{1} x_{0}^{\prime} \gamma_{1}^{\prime}-y_{0}^{\prime} \gamma_{1}+a^{-1} y_{0}^{\prime}\left(q_{1} \gamma_{1}-p_{1} \gamma_{1}^{\prime}\right)-a^{-1} z_{0}^{\prime} p_{1}\right]\right. \\
& +A_{1} k\left[p_{1}\left(1-\gamma_{1}^{\prime 2}\right)+q_{1}\left(1-C_{1}\right) \gamma_{1} \gamma_{1}^{\prime}-S_{2}\left(1+B_{1}\right) \gamma_{1}\right]+0.5 r_{0}^{-1} \ell_{3} p_{1}\left(A^{-1} B_{1}-A_{1} B^{-1}\right) \\
& \left.\times\left[S_{1}+2 z_{0}^{\prime}\left(1-\gamma_{1}^{\prime \prime}\right)-k\left(1-\gamma_{1}^{\prime \prime 2}\right)\right]+A^{-1} r_{0}^{-1} \ell_{3}\left(b^{-1} x_{0}^{\prime}-k b_{1} \gamma_{1}\right) S_{2}\right\} \\
& +\varepsilon^{-3}\left\{0.5 z_{0}^{\prime}\left(a^{-1}-A_{1} b^{-1}\right) \gamma_{1}\left[S_{1}+2 z_{0}^{\prime}\left(1-\gamma_{1}^{\prime \prime}\right)-k\left(1-\gamma_{1}^{\prime \prime 2}\right)\right]\right. \\
& \left.+0.5 A^{-1} r_{0}^{-1} \ell_{3}\left(k B_{1} \gamma_{1}-b^{-1} x_{0}^{\prime}\right)\left[S_{1}+2 z_{0}^{\prime}\left(1-\gamma_{1}^{\prime \prime}\right)-k\left(1-\gamma_{1}^{\prime \prime 2}\right)\right]+p_{1} S_{2}\left(2 k A_{1}-a^{-1} z_{0}^{\prime}\right)\right\}+\cdots, \\
\gamma_{1}+\gamma_{1}= & \varepsilon^{-1}\left[\left(1+B_{1}\right)-B^{-1} r_{0}^{-1} \ell_{3}\right] p_{1} \\
& +\varepsilon^{-2}\left[-S_{1} \gamma_{1}+\left(1+B_{1}\right) p_{1} S_{2}+\left(1-C_{1}\right) p_{1} q_{1} \gamma_{1}^{\prime}+x_{0}^{\prime} \gamma_{1}^{2}+x_{0}^{\prime} b^{-1}-\gamma_{1}\left(y_{0}^{\prime} \gamma_{1}^{\prime}+z_{0}^{\prime} b^{-1}+q_{1}^{2}\right)+k\left(C_{1} \gamma_{1}^{\prime 2}-B_{1}\right) \gamma_{1}^{\prime}\right] \\
& +\varepsilon^{-3}\left[2 b^{-1} x_{0}^{\prime}-\gamma_{1}\left(b^{-1} z_{0}^{\prime}+2 k B_{1}\right)\right] S_{2}+\cdots,
\end{aligned}
$$

where

$$
\begin{aligned}
\omega^{2} & =-A_{1} B_{1}=\frac{(A-C)(B-C)}{A B}=\frac{(a-1)(b-1)}{a b}, \\
\omega^{\prime 2} & =\omega^{2}-\left(A^{-1} B_{1}-A_{1} B^{-1}\right) r_{0}^{-1} \ell_{3} .
\end{aligned}
$$




$$
\begin{aligned}
q_{1}= & A_{1}^{-1} r_{1}^{-1}\left(1-A^{-1} A_{1}^{-1} r_{0}^{-1} \ell_{3} r_{1}^{-1}+\cdots\right) \\
& \cdot\left[-\dot{p}_{1}+\varepsilon^{-1} a^{-1}\left(y_{0}^{\prime} \gamma_{1}^{\prime \prime}-z_{0}^{\prime} \gamma_{1}^{\prime}+k a A_{1} \gamma_{1}^{\prime} \gamma^{\prime \prime}\right)\right], \\
\gamma_{1}^{\prime}= & r_{1}^{-1}\left(\dot{\gamma}_{1}++\varepsilon^{-1} q_{1} \gamma_{1}^{\prime \prime}\right) .
\end{aligned}
$$

Making use of equations (21) and (26) into equations (22) and (23), we obtain a quasi-linear autonomous system with two degrees of freedom and depend on $p_{1}, \dot{p}_{1}, \gamma_{1}, \dot{\gamma}_{1}, p_{10}, \dot{p}_{10}, \gamma_{10}$, and $\dot{\gamma}_{10}$.

Introducing the new variables as follows:

$$
\begin{aligned}
& p_{2}=p_{1}-\varepsilon^{-1}\left(\chi+\chi_{1} \gamma_{2}\right), \\
& \gamma_{2}=\gamma_{1}-\varepsilon^{-1} \nu p_{2},
\end{aligned}
$$

where

$$
\begin{aligned}
\chi= & x_{0}^{\prime}\left(b{\omega^{\prime 2}}^{2}\right)^{-1}\left(A_{1}+A^{-1} r_{0}^{-1} \ell_{3}\right), \\
v= & \left(1-{\omega^{\prime 2}}^{-1}\left[1+B_{1}-B^{-1} r_{0}^{-1} \ell_{3}\right],\right. \\
\chi_{1}= & \left(1-{\omega^{\prime 2}}^{-1}\left[-z_{0}^{\prime}\left(a^{-1}-A_{1} b^{-1}\right)+k\left(A_{1}-\omega^{2}\right)\right.\right. \\
& \left.+A^{-1} r_{0}^{-1} \ell_{3}\left(b^{-1} z_{0}^{\prime}+k B_{1}\right)\right] .
\end{aligned}
$$

Using equations (27), (21), and (26), we obtain

$$
S_{i}=S_{i 1}+2^{2-i} \varepsilon^{-1} S_{i 2}+\cdots, \quad(i=1,2),
$$

where

$$
\begin{aligned}
S_{11}= & a\left(p_{20}^{2}-p_{2}^{2}\right)+b \chi_{3}^{2}\left(\dot{p}_{20}^{2}-\dot{p}_{2}^{2}\right)-2 x_{0}^{\prime}\left(\gamma_{20}-\gamma_{2}\right)-2 y_{0}^{\prime}\left(\dot{\gamma}_{20}-\dot{\gamma}_{2}\right)+k\left[a\left(\gamma_{20}^{2}-\gamma_{2}^{2}\right)+b\left(\dot{\gamma}_{20}^{2}-\dot{\gamma}_{2}^{2}\right)\right], \\
S_{12}= & a\left[\chi\left(p_{20}-p_{2}\right)+\chi_{1}\left(p_{20} \gamma_{20}-p_{2} \gamma_{2}\right)\right]-b \chi_{3}^{2}\left[a^{-1} y_{0}^{\prime}\left(\dot{p}_{20}-\dot{p}_{2}\right)-\chi_{2}\left(\dot{p}_{20} \dot{\gamma}_{20}-\dot{p}_{2} \dot{\gamma}_{2}\right)\right]-v x_{0}^{\prime}\left(p_{20}-p_{2}\right) \\
& -y_{0}^{\prime} \nu_{1}\left(\dot{p}_{20}-\dot{p}_{2}\right)+\left(z_{0}^{\prime}-k\right) S_{21}+k\left[v a\left(p_{20} \gamma_{20}-p_{2} \gamma_{2}\right)+v_{1} b\left(\dot{p}_{20} \dot{\gamma}_{20}-\dot{p}_{2} \dot{\gamma}_{2}\right)\right], \\
S_{21}= & a\left(p_{20} \gamma_{20}-p_{2} \gamma_{2}\right)-b \chi_{3}\left(\dot{p}_{20} \dot{\gamma}_{20}-\dot{p}_{2} \dot{\gamma}_{2}\right), \\
S_{22}= & a\left[v\left(p_{20}^{2}-p_{2}^{2}\right)+\chi\left(\gamma_{20}-\gamma_{2}\right)+\chi_{1}\left(\gamma_{20}^{2}-\gamma_{2}^{2}\right)\right]+b \chi_{3}\left[-v_{1}\left(\dot{p}_{20}^{2}-\dot{p}_{2}^{2}\right)+a^{-1} y_{0}^{\prime}\left(\dot{\gamma}_{20}-\dot{\gamma}_{2}\right)-\chi_{2}\left(\dot{\gamma}_{20}^{2}-\dot{\gamma}_{2}^{2}\right)\right]-\frac{S_{21} \ell_{3}}{\left(C c \sqrt{\gamma_{0}^{\prime \prime}}\right)},
\end{aligned}
$$

where

$$
\begin{aligned}
& \chi_{3}=A_{1}^{-1}\left(1-A^{-1} A_{1}^{-1} r_{0}^{-1} \ell_{3}\right), \\
& \chi_{2}=\chi_{1}+a^{-1} z_{0}^{\prime}-k A_{1}, \\
& v_{1}=v-\chi_{3} .
\end{aligned}
$$

$$
\begin{aligned}
r_{1}= & 1+0.5 \varepsilon^{-2} S_{11}+\varepsilon^{-3}\left(S_{12}-z_{0}^{\prime} S_{21}+k S_{21}\right)+\cdots, \\
\gamma_{1}^{\prime \prime}= & 1+\varepsilon^{-1} S_{21}+\varepsilon^{-2}\left(S_{22}-0.5 S_{11}\right) \\
& -\varepsilon^{-3}\left(S_{12}-z_{0}^{\prime} S_{21}+k S_{21}\right)+\cdots .
\end{aligned}
$$

In terms of $p_{2} \cdot \gamma_{2}$, and the rigid body parameters, we

Formulas (21) and (29) lead to find that

$$
\begin{aligned}
& q_{1}=-\chi_{3} \dot{p}_{2}+\varepsilon^{-1} \chi_{3}\left(a^{-1} y_{0}^{\prime}-\chi_{2} \dot{\gamma}_{2}\right)+\varepsilon^{-2}\left[\chi_{3} \nu_{1} \dot{p}_{2}\left(k A_{1}-a^{-1} z_{0}^{\prime}\right)+S_{11} \dot{p}_{2}\left(\chi_{3}-0.5 A_{1}^{-1}\right)+\chi_{3} S_{21}\left(k A_{1} \dot{\gamma}_{2}+a^{-1} y_{0}^{\prime}\right)\right]+\cdots, \\
& \gamma_{1}^{\prime}=\dot{\gamma}_{2}+\varepsilon^{-1} v_{1} \dot{p}_{2}+\varepsilon^{-2}\left[\chi_{3}\left(a^{-1} y_{0}^{\prime}-\chi_{2} \dot{\gamma}_{2}-S_{21} \dot{p}_{2}\right)-0.5 S_{11} \dot{\gamma}_{2}\right]+\cdots
\end{aligned}
$$

Substituting equations (27), (29), (30), (32), and (33) into equations (23) and (24), we obtain a quasi-linear autonomous system of two degrees of freedom in the following form:

$$
\begin{aligned}
\ddot{p}_{2}+\omega^{\prime 2} p_{2} & =\varepsilon^{-2} F\left(p_{2}, \dot{p}_{2}, \gamma_{2}, \dot{\gamma}_{2}, \varepsilon^{-1}\right), \\
\ddot{\gamma}_{2}+\gamma_{2} & =\varepsilon^{-2} \phi\left(p_{2}, \dot{p}_{2}, \gamma_{2}, \dot{\gamma}_{2}, \varepsilon^{-1}\right),
\end{aligned}
$$




$$
\begin{aligned}
& F=F_{2}+\varepsilon^{-1} F_{3}+\ldots, \phi=\phi_{2}+\varepsilon^{-1} \phi_{3}+\ldots, \\
& F_{2}=f_{2}-\nu \chi_{1}\left(1-\omega^{\prime 2}\right) p_{2}, \phi_{2}=\varphi_{2}+\nu\left(1-\omega^{\prime 2}\right)\left(\chi+\chi_{1} \gamma_{2}\right) \text {, } \\
& F_{3}=f_{2}-\chi_{1} \varphi_{2}-v \chi_{1}\left(1-\omega^{\prime 2}\right)\left(\chi+\chi_{1} \gamma_{2}\right), \phi_{3}=\varphi_{3}-v f_{2}+v^{2} \chi_{1}\left(1-\omega^{\prime 2}\right) p_{2}, \\
& f_{2}=-\omega^{2} S_{11} p_{2}+A_{1} x_{0}^{\prime}\left(b^{-1} S_{21}+\chi_{3} \dot{\gamma}_{2} \dot{p}_{2}\right)+A_{1} C_{1} \chi_{3}^{2} p_{2} \dot{p}_{2}^{2} \\
& -y_{0}^{\prime} \chi_{3} \gamma_{2} \dot{p}_{2}\left(A_{1}+a^{-1}\right)-a^{-1} p_{2}\left(z_{0}^{\prime}+y_{0}^{\prime} \dot{\gamma}_{2}\right)+A_{1} k\left(1-\dot{\gamma}_{2}^{2}\right) p_{2}+\left(C_{1}-1\right) \chi_{3} \gamma_{2} \dot{\gamma}_{2} \dot{p}_{2} \\
& -\left(1+B_{1}\right) S_{21} \gamma_{2}+0.5 r_{0}^{-1} \ell_{3}\left[2 p_{2}\left(A^{-1} B_{1}-A_{1} B^{-1}\right) S_{11}+A^{-1}\left(b^{-1} x_{0}^{\prime}-k B_{1} \gamma_{2}\right) S_{21}\right] \text {, } \\
& f_{3}=-2 \omega^{2} p_{2} S_{12}+\left(\chi+\chi_{1} \gamma_{2}\right)\left\{-\omega^{2} S_{11}-a^{-1}\left(z_{0}^{\prime}+y_{0}^{\prime} \dot{\gamma}_{2}\right)+A_{1}\left[C_{1} \chi_{3}^{2} \dot{p}_{2}^{2}+k\left(1-\dot{\gamma}_{2}^{2}\right)\right]\right\}+A_{1} b^{-1} x_{0}^{\prime} S_{22} \\
& +A_{1} \chi_{3} \dot{p}_{2}\left(x_{0}^{\prime} \nu_{1} \dot{p}_{2}-y_{0}^{\prime} \nu p_{2}\right)-p_{2} \dot{p}_{2}\left[a^{-1} y_{0}^{\prime}\left(\nu_{1}+v \chi\right)+2 A_{1} k v_{1} \dot{\gamma}_{2}\right] \\
& +\chi_{3} \dot{p}_{2}\left(\nu_{1} \gamma_{2} \dot{p}_{2}+\nu \dot{\gamma}_{2} p_{2}\right)\left(C_{1}-1\right)-\left(1-B_{1}\right)\left(\nu S_{21} p_{2}+S_{22} \gamma_{2}\right) \\
& +0.5 z_{0}^{\prime}\left(a^{-1}-A_{1} b^{-1}\right) \gamma_{2} S_{11}+\left(2 k A_{1}-a^{-1} z_{0}^{\prime}\right) p_{2} S_{21}+\chi_{3}\left(a^{-1} y_{0}^{\prime}-\chi_{2} \dot{\gamma}_{2}\right) \\
& \times\left[-A_{1}\left(2 C_{1} \chi_{3} p_{2} \dot{p}_{2}+x_{0}^{\prime} \dot{\gamma}_{2}\right)+y_{0}^{\prime} \gamma_{2}\left(A_{1}+a^{-1}\right)+\gamma_{2} \dot{\gamma}_{2}^{2}\left(1-C_{1}\right)\right] \\
& +0.5 r_{0}^{-1} \ell_{3}\left\{\left(A^{-1} B_{1}-A_{1} B^{-1}\right)\left[2 p_{2}\left(S_{12}-z_{0}^{\prime} S_{21}+k S_{21}\right)+\left(\chi+\chi_{1} \gamma_{2}\right) S_{11}\right]+\left[2 A^{-1}\left[\left(b^{-1} x_{0}^{\prime}-k B_{1} \gamma_{2}\right) S_{22}-k B_{1} \nu S_{21} p_{2}\right]\right.\right. \\
& \left.\left.+A^{-1}\left(k B_{1} \gamma_{2}-b^{-1} x_{0}^{\prime}\right) S_{11}\right]\right\} \text {, } \\
& \varphi_{2}=\left[\left(1+B_{1}\right) S_{21}-\left(1-C_{1}\right) \chi_{3} \dot{\gamma}_{2} \dot{p}_{2}\right] p_{2}+x_{0}^{\prime}\left(b^{-1}+\dot{\gamma}_{2}^{2}\right)+\left[k\left(C_{1} \dot{\gamma}_{2}^{2}-B_{1}\right)-y_{0}^{\prime} \dot{\gamma}_{2}-z_{0}^{\prime} b^{-1}-\chi_{3}^{2} \dot{p}_{2}^{2}-S_{11}\right] \gamma_{2} \text {, } \\
& \varphi_{3}=\left(1+B_{1}\right)\left[p_{2} S_{22}+\left(\chi+\chi_{1} \gamma_{2}\right) S_{21}\right]+\chi_{3}\left(1-C_{1}\right) \times\left\{\left(a^{-1} y_{0}^{\prime}-\chi_{2} \dot{\gamma}_{2}\right) \dot{\gamma}_{2} p_{2}-\dot{p}_{0}\left[v_{1} p_{2} \dot{p}_{2}+\left(\chi+\chi_{1} \gamma_{2}\right) \dot{\gamma}_{2}\right]\right\} \\
& -2 \gamma_{2} S_{12}-v p_{2} S_{11}+2 x_{0}^{\prime} \nu_{1} \dot{\gamma}_{2} \dot{p}_{2}-y_{0}^{\prime}\left(v_{1} \gamma_{2} \dot{p}_{2}+v \dot{\gamma}_{2} p_{2}\right)-v p_{2}\left(b^{-1} z_{0}^{\prime}+\chi_{3}^{2} \dot{p}_{2}^{2}\right)+2 \chi_{3}^{2}\left(a^{-1} y_{0}^{\prime}-\chi_{2} \dot{\gamma}_{2}\right) \times \gamma_{2} \dot{p}_{2} \\
& +k\left[2 C_{1} \nu_{1} \gamma_{2} \dot{\gamma}_{2} \dot{p}_{2}+v\left(C_{1} \dot{\gamma}_{2}^{2}-B_{1}\right) p_{2}\right]+\left[2 b^{-1} x_{0}^{\prime}-\left(b^{-1} z_{0}^{\prime}+2 k B_{1}\right) \gamma_{2}\right] S_{21} \text {. }
\end{aligned}
$$

System (34) has the first integral obtained from equations (17)-(19) as follows:

$$
\gamma_{2}^{2}+\dot{\gamma}_{2}^{2}+2 \varepsilon^{-1}\left(\nu \gamma_{2} p_{2}+v_{1} \dot{\gamma}_{2} \dot{p}_{2}+S_{21}\right)+\varepsilon^{-2}\left[v^{2} p_{2}^{2}+2 \chi_{3} \dot{\gamma}_{2}\left(a^{-1} y_{0}^{\prime}-\chi_{2} \dot{\gamma}_{2}-S_{21} \dot{p}_{2}\right)-\left(1+\dot{\gamma}_{2}^{2}\right) S_{11}+2 S_{22}\right]+\cdots=\left(\gamma_{0}^{\prime \prime}\right)^{-2}-1
$$

We aim to find the periodic solutions for system (36) under the condition $A<B<C\left(\omega^{\prime 2}\right.$ is positive) [12]. In this case, the body rotates about the minor axis of the ellipsoid of inertia surface [13] with initial sufficiently small angular velocity $r_{0}$.

\section{Formal Construction of the Periodic Solutions}

Without a loss of generality, since the system (34) is autonomous, we assume that [14]

$$
\begin{aligned}
p_{2}(0,0) & =0, \\
\dot{p}_{2}(0,0) & =0, \\
\dot{\gamma}_{2}\left(0, \varepsilon^{-1}\right) & =0 .
\end{aligned}
$$

The generating system of equation (34) is

$$
\begin{gathered}
\ddot{p}_{2}^{(0)}+\omega^{\prime 2} p_{2}^{(0)}=0, \\
\ddot{\gamma}_{2}^{(0)}+\gamma_{2}^{(0)}=0,
\end{gathered}
$$

which has periodic solutions as follows: 


$$
\begin{aligned}
& p_{2}^{(0)}=M_{1} \cos \omega^{\prime} \tau+M_{2} \sin \omega^{\prime} \tau, \\
& \gamma_{2}^{(0)}=M_{3} \cos \tau,
\end{aligned}
$$

with a period $T_{0}=2 \pi n$, where $M_{i}, i=(1,2,3)$, are constants to be determined. Consider the required periodic solutions of system (34) in the following form:

$$
\begin{aligned}
p_{2}\left(\tau, \varepsilon^{-1}\right)= & \left(M_{1}+\beta_{1}\right) \cos \omega^{\prime} \tau+\left(M_{2}+\beta_{2}\right) \sin \omega^{\prime} \tau \\
& +\sum_{k=2}^{\infty} \varepsilon^{-k} G_{k}(\tau), \\
\gamma_{2}\left(\tau, \varepsilon^{-1}\right)= & \left(M_{3}+\beta_{3}\right) \cos \tau+\sum_{k=2}^{\infty} \varepsilon^{-k} H_{k}(\tau),
\end{aligned}
$$

with a period $T\left(\varepsilon^{-1}\right)=T_{0}+\alpha\left(\varepsilon^{-1}\right)$. The quantities $\beta_{1}, \omega^{\prime} \beta_{2}$, and $\beta_{3}$ represent the deviations of solutions $p_{2}, \dot{p}_{2}$, and $\gamma_{2}$ at any $\varepsilon$ from their initial values when $\varepsilon \longrightarrow \infty$. Let the initial condition of system (40) be of the following form:

$$
\begin{aligned}
& p_{2}\left(0, \varepsilon^{-1}\right)=M_{1}+\beta_{1}, \\
& \dot{p}_{2}\left(0, \varepsilon^{-1}\right)=\omega^{\prime}\left(M_{2}+\beta_{2}\right), \\
& \gamma_{2}\left(0, \varepsilon^{-1}\right)=M_{3}+\beta_{3}, \\
& \dot{\gamma}_{2}\left(0, \varepsilon^{-1}\right)=0 .
\end{aligned}
$$

Consider the new function as follows:

$$
\begin{aligned}
U= & u+\frac{\partial u}{\partial M_{1}} \beta_{1}+\frac{\partial u}{\partial M_{2}} \beta_{2}+\frac{\partial u}{\partial M_{3}} \beta_{3}+0.5 \frac{\partial^{2} u}{\partial M_{1}^{2}} \beta_{1}^{2}+\cdots \\
& \left(\begin{array}{c}
U=G_{k}, H_{k} \\
u=g_{k}, h_{k}
\end{array}\right),
\end{aligned}
$$

such that

$$
\begin{aligned}
& g_{k}(\tau)=\frac{1}{\omega^{\prime}} \int_{0}^{\tau} F_{k}^{\prime}\left(t_{1}\right) \sin \omega^{\prime}\left(\tau-t_{1}\right) \mathrm{d} t_{1}, \\
& h_{k}(\tau)=\int_{0}^{\tau} \phi_{k}^{\prime}\left(t_{1}\right) \sin \left(\tau-t_{1}\right) \mathrm{d} t_{1} \quad(k=2,3),
\end{aligned}
$$

where

$$
\begin{aligned}
& F_{k}^{\prime}(\tau)=\frac{1}{(k-2) !}\left(\frac{\mathrm{d}^{k-2} F}{\mathrm{~d} \mathcal{\varepsilon}^{2-k}}\right)_{\beta=\varepsilon^{-1}=0}, \\
& \phi_{k}^{\prime}(\tau)=\frac{1}{(k-2) !}\left(\frac{\mathrm{d}^{k-2} \phi}{\mathrm{d} \varepsilon^{2-k}}\right)_{\beta=\varepsilon^{-1}=0} .
\end{aligned}
$$

We note that the right-hand sides of (34) begin with terms of order $\varepsilon^{-2}$ and so

$$
\begin{aligned}
& F_{k}^{\prime}(\tau)=F_{k}\left(p_{2}^{(0)}, \dot{p}_{2}^{(0)}, \gamma_{2}^{(0)}, \dot{\gamma}_{2}^{(0)}\right) \equiv F_{k}^{(0)}, \\
& \phi_{k}^{\prime}(\tau)=\phi_{k}\left(p_{2}^{(0)}, \dot{p}_{2}^{(0)}, \gamma_{2}^{(0)}, \dot{\gamma}_{2}^{(0)}\right) \equiv \phi_{k}^{(0)}, \quad k=2,3 .
\end{aligned}
$$

Now, we find the expressions of $\phi_{k}^{(0)}$ and $F_{k}^{(0)}$. Periodic solutions (39) are reformulated in the following form:

$$
\begin{aligned}
& p_{2}^{(0)}=E \cos \left(\omega^{\prime} \tau-\eta\right), \\
& \gamma_{2}^{(0)}=M_{3} \cos \tau
\end{aligned}
$$

where $E=\sqrt{M_{1}^{2}+M_{2}^{2}}$ and $\eta=\tan ^{-1}\left(M_{2} / M_{1}\right)$.

Using equations (29) and (39), we obtain

$$
\begin{aligned}
S_{i j}^{(0)}= & S_{i j}^{(0)}\left(p_{2}^{(0)}, \dot{p}_{2}^{(0)}, \gamma_{2}^{(0)}, \dot{\gamma}_{2}^{(0)}\right) \quad(i, j=1,2) \\
S_{11}^{(0)}= & E^{2}\left[a\left(\cos ^{2} \eta-0.5\right)+b \chi_{3}^{2} \omega^{\prime 2}\left(\sin ^{2} \eta-0.5\right)+0.5\left(b \chi_{3}^{2} \omega^{\prime 2}-a\right) \cos 2\left(\omega^{\prime} \tau-\eta\right)\right] \\
& -2 M_{3}\left[x_{0}^{\prime}(1-\cos \tau)+y_{0}^{\prime} \sin \tau\right]-0.5 k M_{3}^{2} C_{1}(1-\cos 2 \tau) \\
S_{21}^{(0)}= & M_{3} E\left\{a \cos ^{2} \eta+0.5\left(b \omega^{\prime} \chi_{3}-a\right) \cos \left[\left(\omega^{\prime}-1\right) \tau-\eta\right]-0.5\left(b \omega^{\prime} \chi_{3}+a\right) \cos \left[\left(\omega^{\prime}+1\right) \tau-\eta\right]\right\} \\
S_{12}^{(0)}= & a E\left\{\chi_{3}\left[\cos \eta-\cos \left(\omega^{\prime} \tau-\eta\right)\right]+\chi_{1} M_{3}\left[\cos \eta-\cos \tau \cos \left(\omega^{\prime} \tau-\eta\right)\right]\right\}-b \chi_{3}^{2} E\left[\cos \eta-\cos \left(\omega^{\prime} \tau-\eta\right)\right] \\
& +\chi_{2} M_{3} \sin \tau \sin \left(\omega^{\prime} \tau-\eta\right) \\
& -v x_{0}^{\prime} E\left[\cos \eta-\cos \left(\omega^{\prime} \tau-\eta\right)\right]+k E M_{3}\left\{\nu a\left[\cos \eta-\cos \tau \cos \left(\omega^{\prime} \tau-\eta\right)\right]-v_{1} b \sin \tau \sin \left(\omega^{\prime} \tau-\eta\right)\right\}+\left(z_{0}^{\prime}-k\right) S_{21}^{(0)} \\
S_{22}^{(0)}= & a\left\{\nu E^{2}\left[\cos ^{2} \eta-\cos { }^{2}\left(\omega^{\prime} \tau-\eta\right)\right]+\chi M_{3}(1-\cos \tau)+\chi_{1} M_{3}^{2} \sin ^{2} \tau\right\} \\
& +b \chi_{3}\left\{a^{-1} y_{0}^{\prime} M_{3} \sin \tau-\nu_{1} E^{2} \omega^{\prime 2}\left[\sin ^{2} \eta-\sin ^{2}\left(\omega^{\prime} \tau-\eta\right)\right]+\chi_{2} M_{3}^{2} \sin ^{2} \tau\right\}-S_{21}^{(0)} \ell_{3} /\left(C c \sqrt{\gamma_{0}^{\prime \prime}}\right)
\end{aligned}
$$


Substituting equations (46) and (47) into equation (35), we obtain

$$
\begin{aligned}
F_{2}^{(0)}= & M_{1} L\left(\omega^{\prime}\right) \cos \omega^{\prime} \tau+M_{2} L\left(\omega^{\prime}\right) \sin \omega^{\prime} \tau+\cdots \\
\phi_{2}^{(0)}= & M_{3} N\left(\omega^{\prime}\right) \cos \tau+\cdots \\
L\left(\omega^{\prime}\right)= & \omega^{2}\left[-\left(a M_{1}^{2}+b \omega^{\prime 2} \chi_{3}^{2} M_{2}^{2}\right)+b \omega^{\prime 2} \chi_{3}^{2}\left(M_{1}^{2}+M_{2}^{2}\right)\right] \\
& +A_{1} C_{1} \omega^{\prime 2} \chi_{3}^{2}\left(M_{1}^{2}+M_{2}^{2}\right)+2 M_{3} x_{0}^{\prime} \omega^{2}+k\left(A_{1}+0.5 M_{3}^{2} \omega^{2} C_{1}\right)-\left[z_{0}^{\prime} a^{-1}+v \chi_{1}\left(1-\omega^{\prime 2}\right)\right] \\
& +0.5 r_{0}^{-1} \ell_{3}\left(A^{-1} B_{1}-A_{1} B^{-1}\right)\left[a M_{1}^{2}+b \omega^{\prime 2} \chi_{3}^{2} M_{2}^{2}-b \omega^{\prime 2} \chi_{3}^{2}\left(M_{1}^{2}+M_{2}^{2}\right)-2 M_{3} x_{0}^{\prime}-0.5 k M_{3}^{2} C_{1}\right] \\
N\left(\omega^{\prime}\right)= & -\left(a M_{1}^{2}+b \omega^{\prime 2} \chi_{3}^{2} M_{2}^{2}\right)-\left(M_{1}^{2}+M_{2}^{2}\right)\left[a B_{1}+\omega^{\prime 2} \chi_{3}^{2}(1-b)\right] \\
& \left.+2 M_{3} x_{0}^{\prime}-\left[z_{0}^{\prime} b^{-1}-v \chi_{1}\left(1-\omega^{\prime 2}\right)\right]+k\left(M_{3}^{2} C_{1}-B_{1}\right)\right] .
\end{aligned}
$$

From equations (33), (49), and (50), we obtain

$$
\begin{aligned}
& g_{2}\left(T_{0}\right)=-\pi n\left(\omega^{\prime}\right)^{-1} M_{2} L\left(\omega^{\prime}\right), \dot{g}_{2}\left(T_{0}\right)=\pi n M_{1} L\left(\omega^{\prime}\right), \\
& h_{2}\left(T_{0}\right)=0, \dot{h}_{2}\left(T_{0}\right)=\pi n M_{3} N\left(\omega^{\prime}\right),
\end{aligned}
$$

where the constants $M_{1}, \omega^{\prime} M_{2}$, and $M_{3}$, the deviations $\beta_{1}\left(\varepsilon^{-1}\right), \omega^{\prime} \beta_{2}\left(\varepsilon^{-1}\right)$, and $\beta_{3}\left(\varepsilon^{-1}\right)$, and the correction of the period $\alpha$ are determined from the periodicity conditions and their derivatives:

$$
\begin{aligned}
& \psi_{1}=p_{2}\left(T_{0}+\alpha, \varepsilon^{-1}\right)-p_{2}\left(0, \varepsilon^{-1}\right)=0, \\
& \psi_{2}=\dot{p}_{2}\left(T_{0}+\alpha, \varepsilon^{-1}\right)-\dot{p}_{2}\left(0, \varepsilon^{-1}\right)=0, \\
& \psi_{3}=\gamma_{2}\left(T_{0}+\alpha, \varepsilon^{-1}\right)-\gamma_{2}\left(0, \varepsilon^{-1}\right)=0, \\
& \psi_{4}=\dot{\gamma}_{2}\left(T_{0}+\alpha, \varepsilon^{-1}\right)-\dot{\gamma}_{2}\left(0, \varepsilon^{-1}\right)=0 .
\end{aligned}
$$

Due to the existence of first integral (36) for system (34), the condition $\psi_{3}=0$ is not independent [15]; then, integral (36) becomes

$$
\begin{aligned}
\gamma_{2}^{2}\left(T_{0}\right. & \left.+\alpha, \varepsilon^{-1}\right)+\dot{\gamma}_{2}^{2}\left(T_{0}+\alpha, \varepsilon^{-1}\right)+2 \varepsilon^{-1}\left[\nu \gamma_{2}\left(T_{0}+\alpha, \varepsilon^{-1}\right) p_{2}\left(T_{0}+\alpha, \varepsilon^{-1}\right)+\nu_{1} \dot{\gamma}_{2}\left(T_{0}+\alpha, \varepsilon^{-1}\right) \times \dot{p}_{2}\left(T_{0}+\alpha, \varepsilon^{-1}\right)+S_{21}\left(T_{0}+\alpha, \varepsilon^{-1}\right)\right] \\
& +\varepsilon^{-2}\left\{\nu^{2} p_{2}^{2}\left(T_{0}+\alpha, \varepsilon^{-1}\right)+2 \chi_{3} \dot{\gamma}_{2}\left(T_{0}+\alpha, \varepsilon^{-1}\right)\left[a^{-1} y_{0}^{\prime}-\chi_{2} \dot{\gamma}_{2}\left(T_{0}+\alpha, \varepsilon^{-1}\right)-S_{21}\left(T_{0}+\alpha, \varepsilon^{-1}\right) \dot{p}_{2}\left(T_{0}+\alpha, \varepsilon^{-1}\right)\right]\right. \\
& \left.\left.-S_{11}\left(T_{0}+\alpha, \varepsilon^{-1}\right)\left[1+\dot{\gamma}_{2}^{2}\left(T_{0}+\alpha, \varepsilon^{-1}\right)\right]\right\}+2 S_{22}\left(T_{0}+\alpha, \varepsilon^{-1}\right)\right\}+\ldots \\
= & \gamma_{2}^{2}\left(0, \varepsilon^{-1}\right)+\dot{\gamma}_{2}^{2}\left(0, \varepsilon^{-1}\right)+2 \varepsilon^{-1}\left[\nu \gamma_{2}\left(0, \varepsilon^{-1}\right) p_{2}\left(0, \varepsilon^{-1}\right)+\nu_{1} \dot{\gamma}_{2}\left(0, \varepsilon^{-1}\right) \dot{p}_{2}\left(0, \varepsilon^{-1}\right)+S_{21}\left(0, \varepsilon^{-1}\right)\right] \\
& +\varepsilon^{-2}\left\{\nu^{2} p_{2}^{2}\left(0, \varepsilon^{-1}\right)+2 \chi_{3} \dot{\gamma}_{2}\left(0, \varepsilon^{-1}\right)\left[a^{-1} y_{0}^{\prime}-\chi_{2} \dot{\gamma}_{2}\left(0, \varepsilon^{-1}\right)-S_{21}\left(0, \varepsilon^{-1}\right) \dot{p}_{2}\left(0, \varepsilon^{-1}\right)\right]-S_{11}\left(0, \varepsilon^{-1}\right)\right. \\
& \left.\cdot\left[1+\dot{\gamma}_{2}^{2}\left(0, \varepsilon^{-1}\right)\right]+2 S_{22}\left(0, \varepsilon^{-1}\right)\right\}+\ldots
\end{aligned}
$$

$$
\psi_{1}=\psi_{2}=\psi_{4}=0 \text {. }
$$

Using condition (41) and equation (52), we obtain

$$
\psi_{3}^{2}+2\left(M_{3}+\beta_{3}\right) \psi_{3}+\varepsilon^{-1} \varphi_{1}\left(\psi_{1}, \psi_{2}, \psi_{4}, \varepsilon^{-1}\right)=0,
$$

where $\varphi_{1}$ is an entire function in their variables and $\varphi_{1}\left(0,0,0, \varepsilon^{-1}\right)=0$; then if $M_{3} \neq 0$, form (54) gives

$$
\psi_{3}=f_{1}\left(\psi_{1}, \psi_{2}, \psi_{4}, \varepsilon^{-1}\right) \text {, }
$$

where $f_{1}$ is an entire function in all their arguments and $f_{1}\left(0,0,0, \varepsilon^{-1}\right)=0$; then, the condition $\psi_{3}=0$ in (52) is satisfied with the following condition:
Substituting initial conditions (41) into equation (56) with $\tau=0$, we obtain

$$
M_{3}^{2}+2 M_{3} \beta_{3}+\beta_{3}^{2}+2 \varepsilon^{-1} v M_{3}\left(M_{1}+\beta_{1}\right)+\cdots=\left(\gamma_{0}^{\prime \prime}\right)^{-2}-1 \text {. }
$$

Assume that $\gamma_{0}^{\prime \prime}$ does not depend on $\varepsilon$; we obtain that

$$
\begin{aligned}
M_{3}^{2} & =\left(\gamma_{0}^{\prime \prime}\right)^{-2}-1, \\
\beta_{3}^{2}+2 M_{3} \beta_{3}+2 \varepsilon^{-1} \nu M_{3}\left(M_{1}+\beta_{1}\right)+\cdots & =0 .
\end{aligned}
$$


From (58) and (8), we obtain

$$
\begin{aligned}
& M_{3}=\frac{\sqrt{\left(1-\gamma_{0}^{\prime \prime 2}\right)}}{\gamma_{0}^{\prime \prime}}, \quad 0<M_{3}<\infty, \\
& \beta_{3}=-\varepsilon^{-1} v\left(M_{1}+\beta_{1}\right)+\cdots,
\end{aligned}
$$

where $\gamma_{0}^{\prime \prime}$ is an arbitrary parameter and $M_{3}$ is the arbitrary constant.

This means that periodic solutions (40) depend on arbitrary constant $M_{3}$ and the function $\beta_{3}\left(\varepsilon^{-1}\right)$ which is equal to 0 when $\varepsilon \longrightarrow \infty$. Independent periodic solutions (52) are expanded in a power series of $\alpha$ (neglecting terms of $\varepsilon^{-2} \alpha$ ); then, we obtain

$$
\begin{gathered}
p_{2}\left(T_{0}, \varepsilon^{-1}\right)+\alpha \dot{p}_{2}\left(T_{0}, \varepsilon^{-1}\right)+\ldots=p_{2}\left(0, \varepsilon^{-1}\right), \\
\dot{p}_{2}\left(T_{0}, \varepsilon^{-1}\right)+\alpha \ddot{p}_{2}\left(T_{0}, \varepsilon^{-1}\right)+\ldots=\dot{p}_{2}\left(0, \varepsilon^{-1}\right), \\
\dot{\gamma}_{2}\left(T_{0}, \varepsilon^{-1}\right)+\alpha \ddot{\gamma}_{2}\left(T_{0}, \varepsilon^{-1}\right)+\ldots=\dot{\gamma}_{2}\left(0, \varepsilon^{-1}\right) .
\end{gathered}
$$

Using initial values (41) in the above relations, we put independent periodicity conditions (56) in the following form:

$$
\begin{aligned}
p_{2}\left(T_{0}, \varepsilon^{-1}\right)+\alpha \omega^{\prime}\left(M_{2}+\beta_{2}\right) & =\left(M_{1}+\beta_{1}\right), \\
\dot{p}_{2}\left(T_{0}, \varepsilon^{-1}\right)-\omega^{\prime}\left(M_{2}+\beta_{2}\right) & =\alpha \omega^{\prime}\left(M_{1}+\beta_{1}\right), \\
\dot{\gamma}_{2}\left(T_{0}, \varepsilon^{-1}\right) & =\alpha\left(M_{3}+\beta_{3}\right) .
\end{aligned}
$$

Using equations (40), (59), and the last equation of (61), we obtain the following function:

$$
\alpha\left(\varepsilon^{-1}\right)=\varepsilon^{-2}\left(M_{3}+\beta_{3}\right)^{-1}\left[\dot{H}_{2}\left(T_{0}\right)+\varepsilon \dot{H}_{3}\left(T_{0}\right)+\ldots\right] .
$$

Thus, neglecting the terms of order $\alpha^{2}$ and $\varepsilon^{-2} \alpha$ in (61), we find that the terms of the order $\varepsilon^{-4}$ are neglected. Using equations (37) and (41), we obtain the periodic solutions with basic amplitudes equal to zero, that is [16],

$$
M_{1}=M_{2}=0 .
$$

Substituting equations (62), (63), and (40) into the first two equations from (61), we obtain the following system for determining $\beta_{1}$ and $\beta_{2}$ :

$$
\begin{gathered}
G_{2}\left(T_{0}\right)+\varepsilon^{-1} G_{3}\left(T_{0}\right)+\omega^{\prime} \beta_{2}\left(M_{3}+\beta_{3}\right)^{-1}\left[\dot{H}_{2}\left(T_{0}\right)+\varepsilon^{-1} \dot{H}_{3}\left(T_{0}\right)\right]+\varepsilon^{-2}(\ldots)=0, \\
\dot{G}_{2}\left(T_{0}\right)+\varepsilon^{-1} \dot{G}_{3}\left(T_{0}\right)-\omega^{\prime 2} \beta_{1}\left(M_{3}+\beta_{3}\right)^{-1}\left[\dot{H}_{2}\left(T_{0}\right)+\varepsilon^{-1} \dot{H}_{3}\left(T_{0}\right)\right]+\varepsilon^{-2}(\ldots)=0 .
\end{gathered}
$$

Due to (51), the above system becomes

$$
\begin{array}{r}
-\pi n \beta_{2}\left(\omega^{\prime}\right)^{-1}\left[L_{1}\left(\omega^{\prime}\right)-{\omega^{\prime}}^{2} N_{1}\left(\omega^{\prime}\right)\right]+\varepsilon^{-1}\left[G_{3}\left(T_{0}\right)+\ldots\right]=0, \\
\pi n \beta_{1}\left[L_{1}\left(\omega^{\prime}\right)-\omega^{\prime 2} N_{1}\left(\omega^{\prime}\right)\right]+\varepsilon^{-1}\left[\dot{G}_{3}\left(T_{0}\right)+\ldots\right]=0,
\end{array}
$$

where $L_{1}\left(\omega^{\prime}\right)$ and $N_{1}\left(\omega^{\prime}\right)$ are obtained from (50) replacing $M_{1}, M_{2}, M_{3}$ by $\beta_{1}, \beta_{2}$, and $M_{3}+\beta_{3}$. Making use of equations (24), (28), (31), and (49), we obtain

$$
\begin{aligned}
L_{1}\left(\omega^{\prime}\right)-\omega^{\prime 2} N_{1}\left(\omega^{\prime}\right)= & \left(\beta_{1}^{2}+\beta_{2}^{2}\right) W_{1}\left(\omega^{\prime}\right)+z_{0}^{\prime} W_{2}\left(\omega^{\prime}\right) \\
& +k W_{3}\left(\omega^{\prime}\right)+W_{4}\left(\omega^{\prime}\right),
\end{aligned}
$$

where

$$
\begin{aligned}
W_{1}\left(\omega^{\prime}\right) & =d_{1}+\left(d_{2}+d_{3}\right) r_{0}^{-1} \ell_{3}, \\
W_{2}\left(\omega^{\prime}\right) & =\left(d_{4}-d_{5} d_{6} d_{7}\right)+r_{0}^{-1} \ell_{3}\left[d_{5} d_{6}\left(d_{8}+d_{9}\right)+B^{-1} d_{7}-d_{10}\left(1+a^{-1} d_{6} d_{7}\right)\right], \\
W_{3}\left(\omega^{\prime}\right) & \left.=\left(d_{5} d_{6} d_{11}+d_{12}\right)+r_{0}^{-1} \ell_{3}\left\{d_{5}\left[\left(d_{13}-d_{14}\right)-B^{-1} d_{11}\right]+b^{-1} d_{10}\left(a^{-1} d_{6} d_{11}+d_{15}\right)\right]\right\} r_{0}^{-1} \ell_{3}, \\
W_{4}\left(\omega^{\prime}\right) & =-0.5 a d_{10}\left[\beta_{1}^{2}+\left(\frac{a-1}{b-1}\right) \beta_{2}^{2}\right] r_{0}^{-1} \ell_{3}, \\
d_{1} & =b^{-1}(a-1)(2 a-b-1), \\
d_{2} & =b^{-2}[b(a-b)+(a-1)]\left[a A^{-1}(a-1)(1-b)^{-1}+b B^{-1}\right],
\end{aligned}
$$




$$
\begin{aligned}
& d_{3}=0.5 A^{-1}(1-A)\left[a b^{-1}(1-a)(1-b)^{-1}+A B^{-1}\right], \\
& d_{4}=a^{-1}\left[1-b^{-2}(a-1)(b-1)\right], \\
& d_{5}=(a b)^{-2}[a b+(a-1)(b-1)], \\
& d_{6}=b^{-1}(a+b-1), \\
& d_{7}=(a b)^{-1}(2 b-1)[a b+(a-1)(b-1)], \\
& d_{8}=(A b)^{-1}[a b+(a-1)(b-1)], \\
& d_{9}=(a b)^{-1}(2 b-1)\left[A^{-1} a(a-1)+B^{-1} b(b-1)\right], \\
& d_{10}=(A b)^{-1}(a-1)+(a B)^{-1}(b-1), \\
& d_{11}=(a b)^{-1}(1-b)(a+b-1)[a b+(a-1)(b-1)], \\
& d_{12}=\left(a b^{2}\right)^{-1}(1-b)\left[b^{2}-(a-1)^{2}+0.05 b(a-1)(b-a) M_{3}^{2}\right], \\
& d_{13}=(A b)^{-1}(a-1)[a b+(a-1)(b-1)], \\
& d_{14}=(a b)^{-1}(1-b)(a+b-1)\left[a A^{-1}(a-1)+b B^{-1}(b-1)\right], \\
& d_{15}=0.75 b(b-a) M_{3}^{2}-(a-1) .
\end{aligned}
$$

Since the $z$-axis is oriented towards the minor axis of the ellipsoid of inertia for the body, then $W_{1}\left(\omega^{\prime}\right)>0$ for all $\omega^{\prime}$ under consideration. Assume that [17]

$$
z_{0}^{\prime} W_{2}\left(\omega^{\prime}\right)+k W_{3}\left(\omega^{\prime}\right) \neq 0
$$

Using (65), the expressions for $\beta_{1}$ and $\beta_{2}$ are obtained in the form of power series expansions beginning with terms of order greater than $\varepsilon^{-2}$. So, we obtain the first terms of the required periodic solutions and the correction of the period $\alpha\left(\varepsilon^{-1}\right)$ in the following forms:

$$
\begin{aligned}
p_{1}= & \varepsilon^{-1}\left\{-x_{0}^{\prime}(a-1)^{-1}\left[1+b B^{-1}(a-1)^{-1} r_{0}^{-1} \ell_{3}\right]+\chi_{1} M_{3} \cos \tau\right\}+\cdots \\
q_{1}= & \varepsilon^{-1} a(1-b)^{-1}\left\{y_{0}^{\prime} a^{-1}+\chi_{2} M_{3} \sin \tau-A^{-1}(1-b)^{-1} r_{0}^{-1} \ell_{3}\left[y_{0}^{\prime}+\left(z_{0}^{\prime}-k a A_{1}\right) M_{3} \sin \tau+a d_{5}\left[k b(1-b) d_{6}-z_{0}^{\prime}(2 b-1)\right]\right]\right\}+\cdots \\
r_{1}= & 1-\varepsilon^{-2} M_{3}\left[x_{0}^{\prime}(1-\cos \tau)+y_{0}^{\prime} \sin \tau+0.25 M_{3} C_{1}(1-\cos 2 \tau)\right]+\cdots \\
\gamma_{1}= & M_{3} \cos \tau+\cdots, \\
\gamma_{1}^{\prime}= & -M_{3} \sin \tau+\cdots, \\
\gamma_{1}^{\prime \prime}= & 1+\varepsilon^{-2}\left\{(1-b)^{-1} M_{3} y_{0}^{\prime} \sin \tau+(1-a)^{-1} M_{3} x_{0}^{\prime}(1-\cos \tau)-0.5 b^{-1}(1-b)^{-1} d_{7} M_{3}^{2} z_{0}^{\prime}(1-\cos 2 \tau)\right. \\
& +0.25 M_{3}^{2} k\left(2 a b d_{5} d_{6}+C_{1}\right)(1-\cos 2 \tau) \\
& +r_{0}^{-1} \ell_{3}\left[-a b A^{-1}(1-B)^{-2} M_{3} y_{0}^{\prime} \sin \tau+a b B^{-1}(a-1)^{-2} M_{3} x_{0}^{\prime}(1-\cos \tau)+0.5 b^{-1}(1-b)^{-1} \times z_{0}^{\prime} M_{3}^{2}(1-\cos 2 \tau)\right. \\
& +\left[A^{-1} a^{2} b d_{5}(1-b)^{-1}\left(2 b^{2}-2 b+1\right)+d_{9}\right] \\
& \left.\left.\left.+0.5 k(1-b)^{-1} M_{3}^{2}(1-\cos ) \tau\right)\left[b^{-1} d_{13}-a A^{-1} d_{11}(1-b)^{-1}-(1-b)(2 b-1)^{-1} d_{6} d_{9}\right]\right]\right\}+\cdots \\
& \left.\left.+k\left[d_{6}\left(d_{13}-d_{14}\right)-d_{11} B^{-1}\right]\right]\right\}+\ldots \\
\alpha & \left(\varepsilon^{-1}\right)=\varepsilon^{-2} \pi n\left\{M_{3} x_{0}^{\prime}-z_{0}^{\prime} b^{-1}+(a b)^{-1}\left(k d_{11}-z_{0}^{\prime} d_{7}\right) d_{6}+k\left(M_{3}^{2} C_{1}-B_{1}\right)+(a b)^{-1} r_{0}^{-1} \ell_{3}\left[z_{0}^{\prime}\left[d_{6}\left(d_{8}+d_{9}\right)+d_{7} B^{-1}\right]\right.\right. \\
&
\end{aligned}
$$


New solutions (70)-(76) are obtained in terms of the large parameter $\varepsilon$ and a sufficiently small angular velocity component $r_{o}$ about the minor axis of the ellipsoid of inertia. The case of the motion of the body with a sufficiently small angular velocity component $r_{o}$ about the major axis of the ellipsoid of inertia is considered in a separate paper since $\omega^{\prime 2}$ is negative in this case. The motion considered here is a generalization of many problems studied in a previous work [18]. That is, the obtained solutions give many special cases for gyroscopic problems with new treatment by the large parameter technique [19] which saves high energy given for the body at the initial motion. The correction terms in our solutions in terms of the parameter $\varepsilon$ are

$$
\begin{aligned}
\triangle p_{1}= & \varepsilon^{-1}\left\{x_{0}^{\prime} b^{-1}\left[B_{1}^{-1}\left(1-\omega^{2} \omega^{\prime^{-2}}\right)+\omega^{\prime^{-2}} A^{-1} r_{0}^{-1} \ell_{3}\right]+\left(\chi-\chi_{1}^{*}\right) M_{3} \cos \tau\right\}+\cdots, \\
\triangle q_{1}= & \varepsilon^{-1}\left\{-y_{0}^{\prime}\left(a A A_{1}^{2}\right)^{-1} r_{0}^{-1} \ell_{3}+A_{1}^{-1} M_{3} \sin \tau\left[\chi_{1}-\chi_{1}^{*}-\chi_{2} A^{-1} A_{1}^{-1} r_{0}^{-1} \ell_{3}\right]\right\}+\cdots, \\
\triangle r_{1}= & \varepsilon^{-3}[0]+\ldots, \triangle \gamma_{1}=\varepsilon^{-1}[0]+\ldots, \triangle \gamma_{1}^{\prime}=\varepsilon^{-1}[0]+\cdots, \\
\triangle \gamma_{1}^{\prime \prime}= & \varepsilon^{-2}\left\{a M_{3}\left(\chi-\chi^{*}\right)(1-\cos \tau)-b M_{3} y_{0}^{\prime}\left(a A A_{1}^{2}\right)^{-1} r_{0}^{-1} \ell_{3} \sin \tau\right. \\
& \left.+0.5 M_{3}^{2}(1-\cos 2 \tau)\left[a(1-b)^{-1}\left(\chi_{1}-\chi_{1}^{*}\right)-\chi_{2} b\left(A A_{1}^{2}\right)^{-1} r_{0}^{-1} \ell_{3}\right]\right\}+\cdots, \\
\Delta \alpha\left(\varepsilon^{-1}\right)= & \varepsilon^{-2} \pi n\left\{\left(1-B_{1}\right)\left(\chi_{1}-\chi_{1}^{*}\right)-B^{-1} \chi_{1} r_{0}^{-1} \ell_{3}\right\}+\cdots
\end{aligned}
$$

Also,

$$
\begin{aligned}
\Delta p_{11} & =\Delta p_{1}+\varepsilon^{-1}\left(\chi_{1}^{*}-\chi_{1}^{* *}\right) M_{3} \cos \tau+\cdots, \\
\Delta q_{11} & =\Delta q_{1}+\varepsilon^{-1} A_{1}^{-1} M_{3}\left(\chi_{1}^{*}-\chi_{1}^{* *}-k A_{1}\right) \sin \tau+\cdots, \\
\Delta r_{11} & =-0.25 \varepsilon^{-2} M_{3}^{2} C_{1}(1-\cos 2 \tau)+\cdots, \\
\Delta \gamma_{11} & =\varepsilon^{-1}[0]+\ldots, \Delta \gamma_{11}^{\prime} \\
\Delta \gamma_{11}^{\prime \prime} & =\Delta \gamma_{1}^{\prime \prime}+\varepsilon^{-2}\left\{0.25 k M_{3}^{2} C_{1}(1-\cos 2 \tau)+0.5 M_{3}^{2}(1-\cos \tau)\left[a(1-b)^{-1}\left(\chi_{1}^{*}-\chi_{1}^{* *}\right)-k b\right]\right\}+\cdots, \\
\Delta \alpha_{1}\left(\varepsilon^{-1}\right) & =\Delta \alpha+\varepsilon^{-2} \pi n\left[z_{0}^{\prime}(2-b)^{-1}+k\left(M_{3}^{2} C_{1}-B_{1}\right)+\chi_{1}^{*}\left(1+B_{1}\right)\right]+\cdots,
\end{aligned}
$$

where

$$
\begin{aligned}
\chi^{*} & =A_{1} x_{0}^{\prime}\left(b \omega^{2}\right)^{-1}, \\
\chi_{1}^{*} & =\left(1-\omega^{2}\right)^{-1}\left[k\left(A_{1}-\omega^{2}\right)-z_{0}^{\prime}\left(a^{-1}-A_{1} b^{-1}\right)\right], \\
\chi_{1}^{* *} & =-z_{0}^{\prime}\left(1-\omega^{2}\right)^{-1}\left(a^{-1}-A_{1} b^{-1}\right) .
\end{aligned}
$$

\section{Geometric Interpretation of Motion}

In this section, we explain the geometric interpretation of motion using Euler's angles $\theta, \psi$, and $\varphi[20]$ which are determined from the obtained periodic solutions. Since the initial system is autonomous, the periodic solutions remain so, if the time $t$ is replaced by $\left(t+t_{0}\right)$, where $t_{0}$ is the arbitrary interval time. So, Euler's angles for this problem are given by

$$
\begin{aligned}
\cos \theta & =\gamma^{\prime \prime}, \\
\frac{\mathrm{d} \psi}{\mathrm{d} t} & =\frac{\left(p \gamma+q \gamma^{\prime}\right)}{\left(1-\gamma^{\prime \prime 2}\right)}, \\
\tan \varphi_{0} & =\frac{\gamma_{0}}{\gamma_{0}^{\prime}}, \\
\frac{\mathrm{d} \varphi}{\mathrm{d} t} & =r-\cos \theta\left(\frac{\mathrm{d} \psi}{\mathrm{d} t}\right) .
\end{aligned}
$$

Substituting equations (70)-(76) into equation (80) in which $t$ is replaced by $\left(t+t_{0}\right)$ and using the relations (10), the following expressions for Euler's angles $\theta, \psi$, and $\varphi$ are obtained: 
of the period of the equations of motion of it in the presence of Newtonian force field and an external torque. This problem is solved in a new domain of the angular velocity component $r_{o} \longrightarrow 0$.

The well-known Poincare's method [5] cannot solve this problem because we cannot achieve the small parameter which must be proportional to a sufficiently high angular velocity component $r_{o} \longrightarrow \infty$. So we must search other techniques that come from the sufficiently small assumption of $r_{o}$ and depend on achieving large parameter instead of a small one. This technique is named the large parameter method. The advantage of this method is as follows: assuming low energy at the initial instant instead of high energy, obtaining a slow periodic motion instead of a fast periodic one, and giving the solutions in a new domain of motion $r_{o} \longrightarrow 0$ and $\varepsilon \longrightarrow \infty$. The case when $A<B<\mathrm{C}$ [21] cannot be solved here since $\omega^{\prime 2}$ is negative in this case. So we will treat this case separately in the future, in shaa Allah. The correction terms for our solutions are given in terms of $r_{o}$ and $\varepsilon$. The geometric interpretation of motions is given to describe the orientation of the motion at any instant of time. The cases of gyroscopic motions and regular precession are obtained as special cases from this study when we apply the symmetry conditions. The practical importance of this work is very wide since it is used in many applications of life such as military life and civil one. The case of the gyro motion which is symmetric about the $z$-axis, i.e., $A=B<C$, is obtained as a special case from our work [22]. There are many interesting space applications of these problems in [2].

\section{Data Availability}

The data used to support the findings of this study are available from the corresponding author upon reasonable request.

\section{Conflicts of Interest}

The authors declare that they have no conflicts of interest.

\section{References}

[1] A. V. Borisov and I. S. Mamaev, Rigid Body Dynamics, p. 521, Walter de Gruyter, Berlin, Germany, 2018.

[2] V. S. Aslanov, Rigid Body Dynamics for Space Applications, p. 400, Butter-Worth Heinemann, Oxford, UK, 2017.

[3] F. L. Chernousko, L. D. Akulenko, and D. D. Leshchenko, Evolution of Motions of a Rigid Body About Its Center of Mass, p. 241, Springer, Cham, Switzerland, 2017.

[4] S. V. Ershkov, "A Riccati-type solution of Euler-Poisson equations of rigid body rotation over the fixed point," Acta Mechanica, vol. 228, no. 7, pp. 2719-2723, 2017.

[5] I. A. Arkhangel'skii, "Construction of periodic solutions for the Euler-Poisson equations by means of power series expansion containing a small parameter," Colloquia Mathematica Societatis Janos Bolyai. Differential Equations, pp. 27-50, Elsevier, Keszthely, Hungary, 1975.

[6] A. H. Nayfeh, Introduction to Perturbation Techniques, p. 532, John Wiley \& Sons, New York, NY, USA, 1993.
[7] H. M. Yehia and A. M. Hussein, "New families of integrable two-dimensional systems with quartic second integrals," Nelineinaya Dinamika, vol. 16, no. 2, pp. 211-242, 2020.

[8] G. A. Sahli, "The motion of a rigid body in the presence of a gyrostatic momentum in cases of $l_{3}$ and $l_{3}=0$," Arab Journal of Sciences and Research Publishing, vol. 3, no. 1, p. 22, 2019.

[9] T. S. Amer, A. I. Ismail, and W. S. Amer, "Application of the krylov-bogoliubov-mitropolski technique for a rotating heavy solid under the influence of a gyrostatic moment," Journal of Aerospace Engineering, vol. 25, no. 3, pp. 421-430, 2012.

[10] A. I. Ismail and T. S. Amer, "The fast spinning motion of a rigid body in the presence of a gyrostatic momentum $l_{3}$," Acta Mechanica, vol. 154, no. 1-4, pp. 31-46, 2002.

[11] G. V. Gorr and A. M. Kovalev, The Motion of a Rigid Body, Naukova Dumka, Kyiv, Ukraine, 2013.

[12] J. A. Simon, An Introduction to Lagrangian and Hamiltonian Mechanics, Heriot-Watt University, Edinburgh, Scotland, 2014.

[13] I. A. Arkhangel'skii, "On the motion about a fixed point of a fast spinning heavy solid," Journal of Applied Mathematics and Mechanics, vol. 27, no. 5, pp. 864-877, 1963.

[14] H. M. Yehia, "On the regular precession of an asymmetric rigid body acted upon by uniform gravity and magnetic fields," Egyptian Journal of Basic and Applied Sciences, vol. 2, no. 3, pp. 200-205, 2015.

[15] T. S. Amer, "The rotational motion of the electromagnetic symmetric rigid body," Applied Mathematics \& Information Sciences, vol. 10, no. 4, pp. 1453-1464, 2016.

[16] M. Ehud, Nonlinear Physics of Ecosystems, Taylor \& Francis, Oxford, UK, 2019.

[17] M. Iñarrea, V. Lanchares, A. I. Pascual, and A. Elipe, "Stability of the permanent rotations of an asymmetric gyrostat in a uniform Newtonian field," Applied Mathematics and Computation, vol. 293, pp. 404-415, 2017.

[18] A. A. Elmandouh, "New integrable problems in rigid body dynamics with quartic integrals," Acta Mechanica, vol. 226, no. 8, pp. 2461-2472, 2015.

[19] A. A. Elmandouh, "New integrable problems in the dynamics of particle and rigid body," Acta Mechanica, vol. 226, no. 11, pp. 3749-3762, 2015.

[20] H. M. Yehia and A. A. Elmandouh, "Integrable 2D time-irreversible systems with a cubic second integral," Advances in Mathematical Physics, vol. 2016, Article ID 8958747, 10 pages, 2016.

[21] W. S. Amer, "The necessary and sufficient condition for the stability of a rigid body," Journal of Advances in Physics, vol. 13, no. 4, pp. 4999-5003, 2017.

[22] T. S. Amer and I. M. Abady, "On the solutions of the Euler's dynamic equations for the motion of a rigid body," Journal of Aerospace Engineering, vol. 30, no. 4, 2017. 